\title{
Pulse cyclophosphamide therapy for steroid-resistant focal segmental glomerulosclerosis in children
}

\author{
Abdullah A. Al Salloum, MD
}

Background: In children, steroid-resistant nephritic syndrome due to focal segmental glomerulosclerosis (FSGS) is frequently a progressive condition resulting in end-stage renal disease (ESRD). We report the response of 15 patients with steroid resistant FSGS to treatment with intravenous pulse cyclophosphamide (IVCP) and oral prednisone after 4 years of follow up. Five patients had initial steroid resistance and ten patients had late steroid resistance.

Patients and Methods: All patients were treated with IVCP at a dose of 500 $\mathrm{mg} / \mathrm{m}^{2} / \mathrm{month}$ for 6 months. Adjunctive prednisolone was given at a dose of 60 $\mathrm{mg} / \mathrm{m}^{2} /$ day for 4 weeks followed by $40 \mathrm{mg} / \mathrm{m}^{2} /$ on alternate days for 4 weeks and then tapered over next 4 weeks.

Results: All patients with initial resistance to steroids showed no response to IVCP and continued to be steroid resistant. Three developed CRF during the observation period. The other ten patients with late steroid resistance responded to IVCP, but all were steroid dependent at the end of the observation period. Five could not be weaned from steroids during the IVCP treatment period. The other five patients achieved relatively prolonged remission (7 months to 24 months), but eventually become steroid dependent.

Conclusion: Sixty-seven percent of steroid-resistant FSGS becomes steroid dependent. Patients with initial steroid resistance did not respond to IVCP. We found no correlation between IgM deposition and the response to therapy. The side effects of IVCP were negligible. Beneficial therapy for initial steroid-resistant FSGS remains to be determined.

Key words: Nephrotic syndrome, focal segmental glomerulosclerosis, steroid resistance, pulse cyclophosphamide, children
From the Department of Pediatrics, College of Medicine, King Khalid University Hospital \& King Saud University, Riyadh, Saudi Arabia

Correspondence to:

Abdullah A. Al Salloum, MD

Department of Pediatrics (39)

College of Medicine,

KKUH and King Saud University

P.O. Box 2925

Riyadh 11461

Saudi Arabia

E-mail:asol333@hotmail.com

Accepted for publication: November 2003

Ann Saudi Med 2004; 24(1): 27-30

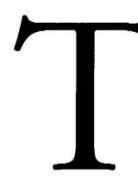
Ireatment of focal segmental glomerulosclerosis (FSGS) is one of the major challenges in the field of pediatric nephrology. The disease is responsible for 10 to 15 percent of pediatric end-stage renal disease (ESRD). ${ }^{1}$ Only about 15 percent of patients with FSGS respond to conventional oral glucocorticoid therapy. The remaining 85 percent, who are steroid resistant, carry a high risk of developing ESRD. ${ }^{2-4}$ The best indicator of outcome of FSGS is the response to steroid therapy. ${ }^{4}$ Many of the patients who responded initially eventually became steroid resistant. ${ }^{5}$ Uncontrolled trials during the past decade have suggested that the disease is not as unresponsive to all treatment as previously thought. ${ }^{4,6}$

Many drug regimens have been used in the treatment of FSGS with variable success. ${ }^{3,5,7-9}$ In what could be described as an extremely aggressive course of treatment, methylprednisolone infusion along with oral prednisone for 12 months was used with complete remission in more than 50 percent of patients. However, this treatment carried a high risk of side effects. ${ }^{3,8}$
The cumulative experience in patients with steroid resistant FSGS treated with cyclosporin A has not been impressive. ${ }^{4}$ Cytotoxic therapy has only rarely been beneficial in patients who are steroid resistant. ${ }^{5}$ Partial response to cyclophosphamide leads to improvement in the clinical outcome of many steroid-resistant nephrotic patients with FSGS.' However, the use of oral cyclophosphamide (OCP) is complicated by attendant side effects. ${ }^{10}$ Intravenous cyclophosphamide (IVCP) has been extensively used in lupus nephritis and various vasculitic disorders and has been shown to be an effective form of therapy with significantly fewer side-effects than OCP. ${ }^{11-13}$ IVCP has been successfully used in steroid-resistant minimal change nephritic syndrome with minimal side effects. ${ }^{14}$ In the present study, we report the response of 15 steroid-resistant FSGS patients to IVCP and the outcome after 4 years of follow up.

\section{Patients and Methods}

The study group was composed of 15 children (10 male, 5 female) who fulfilled the following inclusion criteria: (1) age 
at onset of 1 to 12 years, (2) evidence of steroid resistance by the presence of heavy proteinuria $\left(>40 \mathrm{mg} / \mathrm{m}^{2} / \mathrm{hr}\right)$ despite 4 weeks of standard prednisolone therapy $\left(60 \mathrm{mg} / \mathrm{m}^{2} /\right.$ day), (3) hypoalbuminemia ( $25 \mathrm{mg} / \mathrm{dL}$ ), (4) renal biopsy showing FSGS, (5) absence of identifiable medical diseases associated with FSGS, (6) no prior treatment with cytotoxic or immunosuppressive agents other than steroids, (7) a normal serum creatinine for age, and (8) informed consent provided for IVCP treatment.

The renal biopsies were examined by two independent pathologists. The extent of glomerular sclerosis, interstitial inflammation, interstitial fibrosis and tubular atrophy were determined. Interstitial and tubular changes were described as mild ( $10 \%$ of biopsy material examined), moderate (10$50 \%)$ and severe ( $51 \%)$. The presence of IgM deposits was determined by immunofluorescence. Five patients had been resistant to initial therapy with $60 \mathrm{mg} / \mathrm{m}^{2}$ of daily prednisone administered for 4 weeks. Their proteinuria remained in the nephrotic range with persistent, hypoalbuminemia and edema. They were considered steroid resistant initially. The other ten patients had initially gone into remission within four weeks of $60 \mathrm{mg} / \mathrm{m}^{2}$ daily prednisone therapy, but had suffered from frequent relapses later, and eventually became steroid resistant with nephrotic-range proteinuria while on $60 \mathrm{mg} / \mathrm{m}^{2}$ daily steroid for a further 4 weeks. They were considered late steroid resistant.

The patients were started on monthly infusions of IVCP at a dose of $500 \mathrm{mg} / \mathrm{m}^{2}$ for 6 months. IVCP was administered as an infusion in $250 \mathrm{~mL}$ of normal saline over 3 to 4 hours. A high oral fluid intake was ensured for the following 24 hours to promote frequent voiding of dilute urine to minimize the risk of hemorrhagic cystitis. To minimize bladder toxicity further, the patients received 2-mercaptoethane sulfonate sodium (MESZNA) during the first 12 hours after cyclophosphamide infusion. Adjunctive prednisolone was given in a dose of 60 $\mathrm{mg} / \mathrm{m}^{2} /$ day for 4 weeks followed by $40 \mathrm{mg} / \mathrm{m}^{2} /$ per on alternate days for 4 weeks and then tapered off over the next 4 weeks, unless the patient showed steroid dependency.

The children were followed up monthly for the first 6 months and every 3 months thereafter or whenever medical care was needed. The parents were given instructions to do a urine dipstick test for protein at home once per week. On each visit the child was evaluated clinically for evidence of disease activity and complications. We measured the urine albumin to creatinine ratio in a spot sample, serum albumin, creatinine, hemoglobin and the total leukocyte count. The children were monitored for infections, leucopenia, cataracts, and alopecia. In the presence of infection or leucopenia, the next dose of IVCP was delayed until complete normalization of the count or recovery from infections. The response to IVCP by the end of six months was defined as: (i) complete remission (no or trace proteinuria on urine dipstick, or urine spot albumin to creatinine ratio $<0.2$, increase in serum albumin $>3.5$ $\mathrm{g} / \mathrm{dL}$, complete resolution of edema), (ii) steroid dependency (complete resolution but proteinuria during steroid tapering), (iii) non-response (persistent edema, hypoalbuminemia and proteinuria of $4+$ on urine dipstick or urine spot albumin to creatinine ratio of $>0.2$ by the end of 6 months of IVCP). The proteinuria-free period post IVCP was calculated from the time of completion of IVCP protocol ( $6^{\text {th }}$ dose) to the occurrence of the first relapse. The final outcome after 4 years of observation was classified as (1) sustained remission (complete remission in the last year of observation) (2) frequent relapses (3) steroid dependent (4) non-response (no response to IVCP and patient continue to be steroid resistant). The glomerular filtration rate (GFR) was estimated using the modified height formula of Schwartz. ${ }^{15}$ A GFR $>80 \mathrm{~mL} / \mathrm{min}$ per $1.73 \mathrm{~m}^{2}$ was considered normal.

\section{Results}

The observation period of post-treatment follow-up was 4 years for each patient. None of the patients had renal failure prior to starting IVCP. By the end of the observation period, ten patients were steroid dependent and five patients were steroid resistant (Table 1). All the five patients who had an initial resistance to steroids showed no response to IVCP. They continued to be steroid resistant, with three of them developing CRF during the observation period and one of requiring chronic peritoneal dialysis. Four of the non-responding patients received other alkylating agents and IV methylprednisolone with no response. The fifth patient was given I.V. methyl prednisolone pulses with no response; she was not given other alkylating agents as her renal failure deteriorated rapidly. Her older brother had ESRD secondary to FSGS. There were ten patients who respond to IVCP. The response started after the $2^{\text {nd }}$ dose of IVCP. In this group of responders, five patients achieved relatively prolonged remission $(7$ months to 24 months), but later developed frequent relapses that were treated with oral prednisone similar to the course during IVCP treatment; however, they suffered from frequent relapses and eventually become steroid dependent by the end of the observation period. They were maintained on prednisone $0.5-0.8 \mathrm{mg} / \mathrm{kg}$ per alternate day. The other five patients who responded and became steroid sensitive could not be weaned from the steroid, and continued to be steroid dependent by the end of the four years of observation.

There was no significant difference in the histopathological findings in the group of patients who showed response to IVCP compared to non-responders (Table 1). IgM deposits were positive in only 2 patients of the non-responder group and 5 patients of the responder group. Similarly, there was no significant difference between males and females in response to IVCP. Non-responder patients were of extreme age at presentation. The youngest and oldest (1 year and 8 years) in the group had CRF at the end of the observation period.

The side-effects of cyclophosphamide were minimal. Some patients developed nausea and vomiting in the first 24 
Table 1. Clinical data for 15 children with steroid-resistant focal segmental glomerulosclerosis before and after IVCP therapy.

\begin{tabular}{|c|c|c|c|c|c|c|c|c|c|c|}
\hline \multirow[b]{2}{*}{ No. } & \multirow{2}{*}{$\begin{array}{l}\text { Age at } \\
\text { Onset } \\
\text { (years) }\end{array}$} & \multirow[b]{2}{*}{ Sex } & \multirow{2}{*}{$\begin{array}{l}\text { Steroid } \\
\text { Resistant }\end{array}$} & \multicolumn{4}{|c|}{ Pathology } & \multirow{2}{*}{$\begin{array}{l}\text { Time of } \\
\text { IVCP } \\
\text { response }\end{array}$} & \multirow{2}{*}{$\begin{array}{l}1^{\text {st }} \text { relapse } \\
\text { Post IVCP } \\
\text { (months) }\end{array}$} & \multirow{2}{*}{$\begin{array}{c}\text { Final } \\
\text { Outcome }\end{array}$} \\
\hline & & & & $\begin{array}{l}\text { Glomerular } \\
\text { sclerosis }\end{array}$ & $\begin{array}{c}\text { Interstitial } \\
\text { fibrosis }\end{array}$ & $\begin{array}{l}\text { Tubular, } \\
\text { atrophy }\end{array}$ & $\lg M$ & & & \\
\hline 1 & 2 & $\mathrm{~F}$ & Late & $16 \%$ & Mild & None & $\begin{array}{c}\text { Positive } \\
3+\end{array}$ & $2^{\text {nd do }}$ dose & 8 & Dependen \\
\hline 2 & $21 / 2$ & $M$ & Late & $12 \%$ & Mild & None & Negative & $4^{\text {th }}$ dose & 7 & Dependent \\
\hline 3 & $11 / 2$ & M & Initial & $8 \%$ & Moderate & Mild & $\begin{array}{c}\text { Positive } \\
3+\end{array}$ & - & - & Resistant \\
\hline 4 & 2 & $M$ & Late & $10 \%$ & Mild & Mild & Negative & $3^{\text {rd dose }}$ & Dependent & Dependent \\
\hline 5 & $11 / 2$ & $\mathrm{~F}$ & Initial & $25 \%$ & Mild & Mild & Negative & - & - & $\begin{array}{c}\text { Resistant } \\
\text { (CRF) }\end{array}$ \\
\hline 6 & & $M$ & Initial & $13 \%$ & Mild & None & Negative & - & - & Resistant \\
\hline 7 & 5 & M & Late & $27 \%$ & Moderate & Moderate & $\begin{array}{c}\text { Positive } \\
2+\end{array}$ & $4^{\text {th }}$ dose & 12 & Dependent \\
\hline 8 & $21 / 2$ & M & Late & $10 \%$ & Mild & None & $\begin{array}{c}\text { Positive } \\
2+\end{array}$ & $3^{\text {rd d }}$ dose & Dependent & Dependent \\
\hline 9 & 3 & $M$ & Late & $5 \%$ & Mild & None & $\begin{array}{c}\text { Positive } \\
3+\end{array}$ & $2^{\text {nd }}$ dose & Dependent & Dependent \\
\hline 10 & $11 / 2$ & M & Late & $4 \%$ & Moderate & None & Negative & $2^{\text {nd d }}$ dose & 24 & Dependent \\
\hline 11 & 6 & $\mathrm{~F}$ & Late & $9 \%$ & Mild & None & $\begin{array}{c}\text { Positive } \\
3+\end{array}$ & $2^{\text {nd }}$ dose & Dependent & Dependent \\
\hline 12 & 8 & $\mathrm{M}$ & Initial & $14 \%$ & Mild & Mild & $\begin{array}{c}\text { Positive } \\
2+\end{array}$ & - & - & $\begin{array}{l}\text { Resistant } \\
\text { (CRF) }\end{array}$ \\
\hline 13 & $11 / 2$ & $M$ & Late & $12 \%$ & Mild & None & Negative & $4^{\text {th }}$ dose & Dependent & Dependent \\
\hline 14 & 1 & $\mathrm{~F}$ & Initial & $8 \%$ & Mild & None & Negative & - & - & $\begin{array}{c}\text { Resistant } \\
\text { (CRF) }\end{array}$ \\
\hline 15 & 3 & $\mathrm{~F}$ & Late & $14 \%$ & Mild & None & Negative & $3^{\text {rd dose }}$ & 12 & Dependent \\
\hline
\end{tabular}

hours post-IVCP infusion, which subsided easily with antiemetic therapy. None of the children developed leucopenia, hemorrhagic cystitis or alopecia. One patient in the IVCP resistant group developed bilateral cataracts and required surgery.

\section{Discussion}

Although alkylating agents, specifically cyclophosphamide and chlorambucil, have minimal efficacy in children with FSGS who have shown resistance to glucocorticoid treatment, ${ }^{5}$ they still widely used as an alternative to or in combination with glucocorticoids. ${ }^{6}$ They spare the patient from large cumulative doses of glucocorticoid, which provoke unwanted adverse effects. ${ }^{3}$ It is widely believed that concurrent use of an alkylating agent during glucocorticoid treatment increases the probability of inducing remission. ${ }^{4}$ Most authors agree that the duration of remission induced by an alkylating agent is roughly proportional to the length of the course of treatment. ${ }^{14}$ However, the potential side effects of these drugs limit their use. Leucopenia, chemical cystitis, alopecia and sterility pose considerable problems in patients exposed to prolonged courses of OCP., ${ }^{2,4}$ A combination of pulse methylprednisolone and OCP has been advocated, but prolonged patient hospitalization and steroid side effects such as hypertension, cataract, and growth retardation limit their use. ${ }^{2-4,7}$ In IVCP-induced and sustained remission in children with steroid-resistant minimal change nephrotic syndrome, one study showed a better outcome than prolonged treatment with OCP. ${ }^{14}$ The total IVCP dose used was lower than the oral dose at $2 \mathrm{mg} / \mathrm{kg}$ daily for 8 weeks. The author did not report any adverse effect of IVCP therapy. Rennert et $\mathrm{al}^{16}$ reported the use of IVCP in ten patients with steroid resistant FSGS who had promising results as sustained remission was achieved in 70 percent of the patients. However, the follow-up period was short.

In our study, IVCP was found to be effective in late responders. The outcome in initially steroid-resistant patients was poor; none of the five patients responded to IVCP and three progressed to renal failure. This result is similar to the outcome in patients who used OCP. ${ }^{4,5}$ We could not determine a predictive value of the percentage of glomeruli with segmental sclerosis in terms of response to IVCP and final outcome. The presence of IgM deposits on the biopsy specimen were not related to the eventual response to the treatment. This support the results of other studies that could not find clear correlations between the extent of sclerosis or IgM deposition and progression of FSGS or its response to therapy. ${ }^{2,4}$

With this modality of treatment, 67 percent of our patients who were steroid resistant before IVCP become steroid dependent. This improvement in the clinical outcome 
may help patients with steroid-resistant FSGS. The cumulative doses given were far below those considered toxic. The side effects of IVCP were negligible. Patients with initial steroid resistance did not respond. Beneficial therapy for this group of patients remains to be determined.

\section{Acknowledgment}

The author would like to thank Prof. N. Al Jurayyan, Prof. S. Al Rasheed (Pediatric Department), Prof. M. Al Sohaibani, Dr. A. Al Rikabi (Pathology Department) for their valuable contribution and advice.

\section{References}

1. Warady BA, Hebert D, Sullivan $K E$, Alexander $S R$, Tegani A. Renal transplantation, chronic dialysis and chronic renal insufficiency in children and adolescents. The 1995 annual report of the North American Pediatric Renal Transplant Cooperative Study. Pediatr Nephrol. 1997;11: 49-64.

2. Ichikawal, FogoA. Focal Segmental Glomerulosclerosis. Pediatr Nephrol. 1996;10: 374-391.

3. Mendoza S, Reznik VM, GriswoldWR, KrenskyAM, Yorgin $P D$, Tune BM. Treatment of steroid resistant focal segmental glomerulosclerosis with pulse methylprednisolone and alkylating agents. Pediatr Nephrol. 1990;4: 303.

4. Korbet SM, Schwartz MM, Lewis EJ. Primary focal segmental glomerulosclerosis: clinical course and response to therapy. Am J Kidney Dis. 1994;23: 773-783.

5. Tarshish P, Tobin JN, Bernstein J, Edelmann JR. CM cyclophosphamide does not benefit patients with focal segmental glomerulosclerosis. A report of the international study of kidney diseases in children. Pediatr Nephrol. 1996; 10: 590-593.
6. Vehaskari VM. Treatment practices of FSGS among Northem American Pediatric Nephrologist. Pediatr Nephrol. 1999;13: 301-303.

7. Tune BM, Lieberman E, Mendoza SA (1996). Steroid resistant nephrotic focal segmental glomerulosclerosis: a treatable disease. Pediatr Nephrol. 1996;10: 772 778.

8. Aviles DH, Irwin KC, Dublin LS, Vehaskari VM Aggressive treatment of severe idiopathic focal segmental glomerulosclerosis. Pediatr Nephrol. 1996;13: 298-300.

9. Geary DF, Favine M, Thorner P, Baumal R. Response to cyclophosphamide in steroid resistant focal segmental glomeruloclerosis: a reappraisal. Clin Nephrol. 1984; 22: 109-113.

10. Gulati S, Pokhariyal S, Sharma RK, Elhence R, Kher V, Pandey CM, Gupta A. Pulse cyclophosphamide therapy in frequently relapsing nephrotic syndrome. Nehprol Dial Transplant. 2001;16: 2013-2017.

11. Austin HA, Klippel JH, Balow JE, Le Riche NG Steinberg AD, Plotz PH, Decken JL. Therapy of lupus nephritis controlled trial of prednisolone and cytotoxic drugs. N Engl J Med. 1986;314: 614-619.

12. Martin F, Lanwerys $B$, Lefbuz C, Devogelair JP, Houssain FA. Side effects of intravenous cyclophosphamide pulse. Therap Lupus. 1997;6: 254-257.

13. Haubitz $M$, Ehlerding C, Kamino A, Koch KM Brunkhorst R. Reduced gonadal toxicity after IV cyclophosphamide administration in patients with non malignant disease. Clin Nephrol. 1988;49: 19-23.

14. Elhence R, Gulati S, Kher V, Gupta A, Sharma RK. Intravenous pulse cyclophosphamide: a new regimen for steroid resistant minimal change nephrotic syndrome. Pediatr Nephrol. 1994;8: 1-3.

15. Schwartz GJ, Brion LP, Spitzer A. The use of plasma creatinine concentration for estimating glomerular filtration rate in infants, children and adolescents. Pediatr Clin North Am. 1987; 34: 571-590.

16. Rennert WP, Kulu UK, Jacobs D, Goetsch S, Verhaart S. Pulse cyclophosphamide for steroid resistant focal segmental glomerulosclerosis. Pediatr Nephrol. 1999;13:113-116. 